\title{
TAUBERIAN CONDITIONS FOR A GENERAL LIMITABLE METHOD
}

\author{
İBRAHIM C̣ANAK AND ÜMİT TOTUR
}

Received 16 July 2006; Revised 21 August 2006; Accepted 21 August 2006

Let $\left(u_{n}\right)$ be a sequence of real numbers, $L$ an additive limitable method with some property, and $\mathcal{U}$ and $\mathscr{V}$ different spaces of sequences related to each other. We prove that if the classical control modulo of the oscillatory behavior of $\left(u_{n}\right)$ in $\boldsymbol{U}$ is a Tauberian condition for $L$, then the general control modulo of the oscillatory behavior of integer order $m$ of $\left(u_{n}\right)$ in $\mathcal{U}$ or $\mathscr{V}$ is also a Tauberian condition for $L$.

Copyright (c) 2006 Hindawi Publishing Corporation. All rights reserved.

\section{Introduction}

In this paper, $u_{n}=O(1)$ and $u_{n}=o(1)$ denote $O(1)$ as $n \rightarrow \infty$ and $o(1)$ as $n \rightarrow \infty$, respectively. Let $\mathcal{N}, \mathscr{B}, \mathscr{Y}$, and $\mathcal{M}$ denote the space of sequences converging to 0 , bounded, slowly oscillating, and moderately oscillating, respectively.

The classical control modulo of the oscillatory behavior of $\left(u_{n}\right)$ is denoted by $\omega_{n}^{(0)}(u)=$ $n \Delta u_{n}$ and the general control modulo of the oscillatory behavior of order $m$ of $\left(u_{n}\right)$ is defined by $\omega_{n}^{(m)}(u)=\omega_{n}^{(m-1)}(u)-\sigma_{n}^{(1)}\left(\omega^{(m-1)}(u)\right)$, where

$$
\Delta u_{n}=\left\{\begin{array}{ll}
u_{n}-u_{n-1}, & n \geq 1, \\
u_{0}, & n=0,
\end{array} \sigma_{n}^{(1)}(u)=\frac{1}{n+1} \sum_{k=0}^{n} u_{k} .\right.
$$

Tauber [10] proved that if $\left(u_{n}\right)$ is Abel limitable and

$$
\left(\omega_{n}^{(0)}(u)\right) \in \mathcal{N}
$$

then $\left(u_{n}\right)$ is convergent. The condition (1.2) on the sequence $\left(u_{n}\right)$ is called a Tauberian condition for Abel limitable method and the resulting theorem is called a Tauberian theorem. 
2 Tauberian conditions for a general limitable method

Tauber [10] further proved that the condition

$$
\left(\sigma_{n}^{(1)}\left(\omega^{(0)}(u)\right)\right) \in \mathcal{N}
$$

is also a Tauberian condition. It was shown by Littlewood [6] that the condition (1.2) could be replaced by

$$
\left(\omega_{n}^{(0)}(u)\right) \in \mathscr{B}
$$

Hardy and Littlewood [5] improved Littlewood's theorem replacing (1.4) by onesided boundedness of $\left(\omega_{n}^{(0)}(u)\right)$.

Stanojević [9] reformulated the definition of slow oscillation given by Schmidt [8] in a more suitable form and then proved that the conditions (1.2) and (1.3) could be replaced by

$$
\begin{gathered}
\left(\omega_{n}^{(0)}(u)\right) \in \mathscr{Y}, \\
\left(\sigma_{n}^{(1)}\left(\omega^{(0)}(u)\right)\right) \in \mathscr{S},
\end{gathered}
$$

respectively.

A generalization of slow oscillation, moderate oscillation, was introduced by Stanojević and it was proved by Dik [4] that (1.5) could be replaced by

$$
\left(\omega_{n}^{(0)}(u)\right) \in M
$$

and (1.6) could not be replaced by

$$
\left(\sigma_{n}^{(1)}\left(\omega^{(0)}(u)\right)\right) \in \mathcal{M} .
$$

Recently, Canak and Totur [3] have shown that for any nonnegative integer $m \geq 1$,

$$
\left(\omega_{n}^{(m)}(u)\right) \in \mathcal{M}
$$

is a Tauberian condition for Abel limitable method.

Meyer-König and Tietz [7] proved that if (1.2) is a Tauberian conditions for an additive and regular limitability method, then (1.3) is a Tauberian condition for L. Canak et al. [1] extended and generalized Meyer-König and Tietz's [7] result and obtained the following theorems for an additive and $(C, 1)$ regular method $L$.

Theorem 1.1. If $\left(\omega_{n}^{(0)}(u)\right) \in \mathscr{S}$ is a Tauberian condition for an additive and $(C, 1)$ regular limitable method $L$, then $\left(\omega_{n}^{(1)}(u)\right) \in \mathscr{S}$ is a Tauberian condition for $L$.

Theorem 1.2. If $\left(\omega_{n}^{(0)}(u)\right) \in \mathscr{B}$ is a Tauberian condition for an additive and $(C, 1)$ regular limitable method $L$, then $\left(\omega_{n}^{(1)}(u)\right) \in \mathscr{B}$ is a Tauberian condition for $L$.

Let $\mathcal{U}$ and $\mathscr{V}$ be distinct spaces of sequences related to each other. In this paper, we prove that if the classical control modulo of the oscillatory behavior of $\left(u_{n}\right)$ in $u$ is a Tauberian condition for an additive and $(C, 1)$ limitable method $L$, then the general control modulo of the oscillatory behavior of integer order $m$ of $\left(u_{n}\right)$ in $\mathcal{u}$ or $\mathscr{V}$ is also a Tauberian condition for $L$. 


\section{Notations and definitions}

Throughout this paper, let $u=\left(u_{n}\right)$ be a sequence of real numbers. For each integer $m \geq 0$ and for all nonnegative integers $n$ denote $\sigma_{n}^{(m)}(u)$ by

$$
\sigma_{n}^{(m)}(u)= \begin{cases}\frac{1}{n+1} \sum_{k=0}^{n} \sigma_{k}^{(m-1)}(u)=u_{0}+\sum_{k=1}^{n} \frac{V_{k}^{(m-1)}(\Delta u)}{k}, & m \geq 1, \\ u_{n}, & m=0,\end{cases}
$$

where

$$
V_{n}^{(m)}(\Delta u)= \begin{cases}\sigma_{n}^{(1)}\left(V^{(m-1)}(\Delta u)\right), & m \geq 1 \\ \frac{1}{n+1} \sum_{k=0}^{n} k \Delta u_{k}, & m=0\end{cases}
$$

The identity

$$
u_{n}-\sigma_{n}^{(1)}(u)=V_{n}^{(0)}(\Delta u)
$$

is well known and will be extensively used. We define inductively for each integer $m \geq 1$ and for all nonnegative integers $n$,

$$
(n \Delta)_{m} u_{n}=n \Delta\left((n \Delta)_{m-1} u_{n}\right), \quad \text { where }(n \Delta)_{0} u_{n}=u_{n}
$$

It is proved in [2] that for each integer $m \geq 1$,

$$
\omega_{n}^{(m)}(u)=(n \Delta)_{m} V_{n}^{(m-1)}(\Delta u)
$$

Definition 2.1. A sequence $u=\left(u_{n}\right)$ is Abel limitable to $s$ if the $\operatorname{limit}_{\lim _{x \rightarrow 1^{-}}}(1-$ x) $\sum_{n=0}^{\infty} u_{n} x^{n}=s$.

Definition 2.2. A sequence $u=\left(u_{n}\right)$ is $L$ limitable to $s$ if $L-\lim _{n} u_{n}=s$.

A limitation method $L$ is called additive if $L-\lim _{n} u_{n}=s$ and $L-\lim _{n} v_{n}=t$ imply that $L-\lim _{n}\left(u_{n}+v_{n}\right)=s+t$. A limitation method $L$ is called regular if the $L-$ limit of every convergent sequence is equal to its limit. $L$ is called $(C, 1)$ regular if $L-\lim _{n} u_{n}=s$ implies $L-\lim _{n} \sigma_{n}^{(1)}(u)=s$. It is clear that every regular limitable method is $(C, 1)$ regular. Definition 2.3. A sequence $u=\left(u_{n}\right)$ is one-sidedly bounded if for some $C \geq 0$ and for each nonnegative integer $n, u_{n} \geq-C$.

Definition 2.4. A sequence $u=\left(u_{n}\right)$ is slowly oscillating [9] if

$$
\lim _{\lambda \rightarrow 1^{+}} \varlimsup_{n} \max _{n+1 \leq k \leq[\lambda n]}\left|\sum_{j=n+1}^{k} \Delta u_{j}\right|=0,
$$

where $[\lambda n]$ denotes the integer part of $\lambda n$. 
4 Tauberian conditions for a general limitable method

A sequence $u=\left(u_{n}\right) \in \mathscr{S}$ if and only if $\left(V_{n}^{(0)}(\Delta u)\right) \in \mathscr{S}$ and $\left(V_{n}^{(0)}(\Delta u)\right) \in \mathscr{B}$ (see [4]). The next definition is a generalization of slow oscillation.

Definition 2.5. A sequence $u=\left(u_{n}\right)$ is moderately oscillating [9] if for $\lambda>1$,

$$
\varlimsup_{n} \max _{n+1 \leq k \leq[\lambda n]}\left|\sum_{j=n+1}^{k} \Delta u_{j}\right|<\infty .
$$

A sequence $\left(u_{n}\right) \in \mathcal{M}$ if and only if $\left(V_{n}^{(0)}(\Delta u)\right) \in \mathscr{B}$ (see [4]).

\section{Results and proofs}

Theorem 3.1. If $\left(\omega_{n}^{(0)}(u)\right) \in M$ is a Tauberian condition for $L$, then for any integer $m \geq 1$, $\left(\omega_{n}^{(m)}(u)\right) \in M$ is also a Tauberian condition for $L$.

Proof. Assume that $\left(\omega_{n}^{(0)}(u)\right) \in \mathcal{M}$ is a Tauberian condition for $L$. Let $L-\lim _{n} u_{n}=s$. Since $L$ is $(C, 1)$ regular, it follows by $(2.3)$ that $L-\lim _{n} V_{n}^{(0)}(\Delta u)=0$. It is obvious that $L-\lim _{n} u_{n}=s$ implies $L-\lim _{n}(n \Delta)_{m-1} V_{n}^{(m-1)}(\Delta u)=0$. Since

$$
\left(\omega_{n}^{(m)}(u)\right)=\left(n \Delta\left((n \Delta)_{m-1} V_{n}^{(m-1)}(\Delta u)\right)\right) \in \mathcal{M}
$$

by assumption, we have

$$
(n \Delta)_{m-1} V_{n}^{(m-1)}(\Delta u)=o(1)
$$

By the same reasoning, we deduce that

$$
(n \Delta)_{m-1} V_{n}^{(m-1)}(\Delta u)=n \Delta\left((n \Delta)_{m-2} V_{n}^{(m-1)}(\Delta u)\right)=o(1)
$$

and $L-\lim _{n}(n \Delta)_{m-2} V_{n}^{(m-1)}(\Delta u)=0$. Again by assumption, we have

$$
(n \Delta)_{m-2} V_{n}^{(m-1)}(\Delta u)=o(1)
$$

From the identity

$$
(n \Delta)_{m-1} V_{n}^{(m-1)}(\Delta u)=(n \Delta)_{m-2} V_{n}^{(m-2)}(\Delta u)-(n \Delta)_{m-2} V_{n}^{(m-1)}(\Delta u),
$$

(3.2), and (3.4), we have

$$
(n \Delta)_{m-2} V_{n}^{(m-2)}(\Delta u)=o(1)
$$

Continuing in this vein, we have

$$
n \Delta V_{n}^{(1)}(\Delta u)=o(1)
$$

Since $L-\lim _{n} V_{n}^{(1)}(\Delta u)=0$, it follows by (3.7) that

$$
V_{n}^{(1)}(\Delta u)=o(1)
$$


From (3.7) and (3.8), we have $V_{n}^{(0)}(\Delta u)=o(1) . L-\lim _{n} \sigma_{n}^{(1)}(u)=s$ and $V_{n}^{(0)}(\Delta u)=$ $n \Delta \sigma_{n}^{(1)}(u)=o(1)$ imply that $\lim _{n} \sigma_{n}^{(1)}(u)=s$. Hence, by $(2.3),\left(u_{n}\right)$ converges to $s$.

Theorem 3.2. If $\left(\omega_{n}^{(0)}(u)\right) \in \mathscr{B}$ is a Tauberian condition for $L$, then for any integer $m \geq 1$, $\left(\omega_{n}^{(m)}(u)\right) \in \mathscr{B}$ is also a Tauberian condition for $L$.

Proof. Assume that $\omega_{n}^{(0)}(u)=O(1)$ is a Tauberian condition for $L$. Let $L-\lim _{n} u_{n}=s$. Since $L-\lim _{n}(n \Delta)_{m-1} V_{n}^{(m-1)}(\Delta u)=0$ and $\omega_{n}^{(m)}(u)=n \Delta\left((n \Delta)_{m-1} V_{n}^{(m-1)}(\Delta u)\right)=O(1)$, $(n \Delta)_{m-1} V_{n}^{(m-1)}(\Delta u)=o(1)$ by assumption. The rest of the proof is as in the proof of Theorem 3.1.

Theorem 3.3. If for some $C \geq 0, \omega_{n}^{(0)}(u) \geq-C$ is a Tauberian condition for $L$, then for any integer $m \geq 1, \omega_{n}^{(m)}(u) \geq-C$ is also a Tauberian condition for $L$.

Proof. Assume that $\omega_{n}^{(0)}(u) \geq-C$ for some $C \geq 0$ is a Tauberian condition for $L$. Let $L-\lim _{n} u_{n}=s$. Since $L-\lim _{n}(n \Delta)_{m-1} V_{n}^{(m-1)}(\Delta u)=0$ and $\omega_{n}^{(m)}(u)=n \Delta\left((n \Delta)_{m-1} V_{n}^{(m-1)}\right.$ $(\Delta u)) \geq-C,(n \Delta)_{m-1} V_{n}^{(m-1)}(\Delta u)=o(1)$ by assumption. The rest of the proof is as in the proof of Theorem 3.1 .

We now prove that if $\left(\omega_{n}^{(0)}(u)\right) \in \mathcal{M}$ (or $\in \mathscr{B}$ ) is a Tauberian condition for $L$, then for any integer $m \geq 1,\left(\omega_{n}^{(m)}(u)\right) \in \mathscr{S}$ (or $\in \mathcal{M}$ ) is a Tauberian condition for $L$, respectively.

Theorem 3.4. If $\left(\omega_{n}^{(0)}(u)\right) \in \mathcal{M}$ is a Tauberian condition for $L$, then for any integer $m \geq 1$, $\left(\omega_{n}^{(m)}(u)\right) \in \mathscr{B}$ is also a Tauberian condition for $L$.

Proof. It is sufficient to note that $\omega_{n}^{(m)}(u)=(n \Delta)_{m} V_{n}^{(m-1)}(\Delta u)=V_{n}^{(0)}\left(\Delta \omega^{(m-1)}(u)\right)$ $=O(1)$ implies $\left(\omega_{n}^{(m-1)}(u)\right) \in \mathcal{M}$. Proof now follows from Theorem 3.1.

THeOREM 3.5. If $\left(\omega_{n}^{(0)}(u)\right) \in \mathscr{B}$ is a Tauberian condition for $L$, then for any integer $m \geq 1$, $\left(\omega_{n}^{(m)}(u)\right) \in \mathcal{M}$ is also a Tauberian condition for $L$.

Proof. It is sufficient to note that $\left(\omega_{n}^{(m)}(u)\right) \in \mathcal{M}$ implies $V_{n}^{(0)}\left(\Delta \omega^{(m)}(u)\right)=\omega_{n}^{(m+1)}(u)=$ $O(1)$. Proof now follows from Theorem 3.4.

Remark 3.6. Because of the inclusion $\mathcal{N} \subset \mathscr{S} \subset \mathcal{M}$, the condition "belonging to $\mathcal{M}$ " can be replaced by "belonging to $\mathscr{S}$ " or "belonging to $\mathcal{N}$."

In Theorems 3.1, 3.2, and 3.3, taking $m=1$ and replacing $\mathcal{M}$ by $\mathscr{Y}$, we have $[1$, Theorems 4.1, 4.2, and 4.4] by Canak et al.

\section{Acknowledgment}

This research was supported by Adnan Menderes University under Grant FEF-06011.

\section{References}

[1] İ. Çanak, M. Dik, and F. Dik, On a Theorem of W. Meyer-König and H. Tietz, International Journal of Mathematics and Mathematical Sciences 2005 (2005), no. 15, 2491-2496.

[2] İ. Çanak and Ü. Totur, A Tauberian theorem with a generalized one-sided condition, preprint, 2005. 


\section{Tauberian conditions for a general limitable method}

[3] __ Tauberian theorems for Abel limitable sequences with controlled oscillatory behavior, preprint, 2006.

[4] M. Dik, Tauberian theorems for sequences with moderately oscillatory control modulo, Mathematica Moravica 5 (2001), 57-94.

[5] G. H. Hardy and J. E. Littlewood, Tauberian theorems concerning power series and Dirichlet's series whose coeffients are positive, Proceedings of the London Mathematical Society 2 (1914), no. 13, 174-191.

[6] J. E. Littlewood, The converse of Abel's theorem on power series, Proceedings of the London Mathematical Society 2 (1911), no. 9, 434-448.

[7] W. Meyer-König and H. Tietz, On Tauberian conditions of type o, Bulletin of the American Mathematical Society 73 (1967), 926-927.

[8] R. Schmidt, Über divergente Folgen und lineare Mittelbildungen, Mathematische Zeitschrift 22 (1925), no. 1, 89-152.

[9] Č. V. Stanojević, Analysis of Divergence: Control and Management of Divergent Processes, edited by İ. C̣anak, Graduate Research Seminar Lecture Notes, University of Missouri-Rolla, Missouri, 1998.

[10] A. Tauber, Ein Satz aus der Theorie der unendlichen Reihen, Monatshefte für Mathematik 8 (1897), 273-277.

İbrahım C̣anak: Department of Mathematics, Adnan Menderes University, 09010 Aydin, Turkey E-mail address: icanak@adu.edu.tr

Ümıt Totur: Department of Mathematics, Adnan Menderes University, 09010 Aydin, Turkey

E-mail address: utotur@adu.edu.tr 


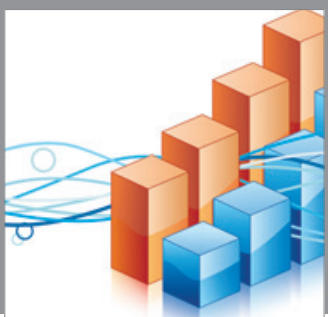

Advances in

Operations Research

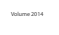

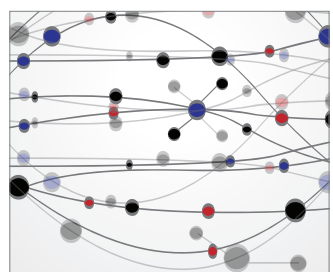

\section{The Scientific} World Journal
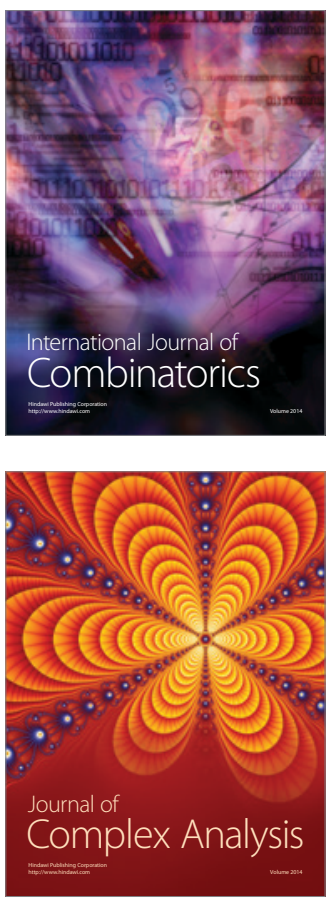

International Journal of

Mathematics and

Mathematical

Sciences
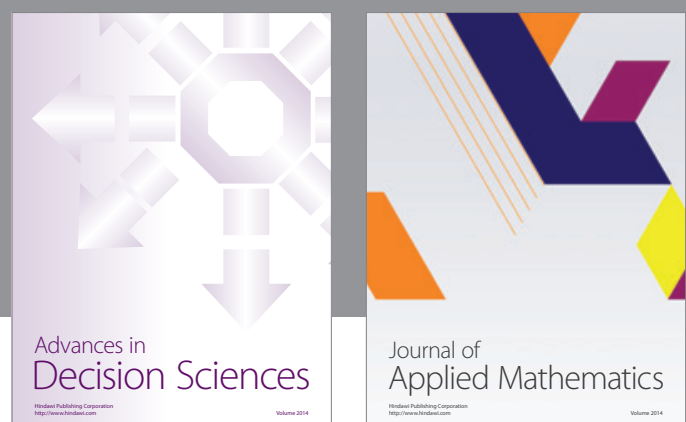

Journal of

Applied Mathematics
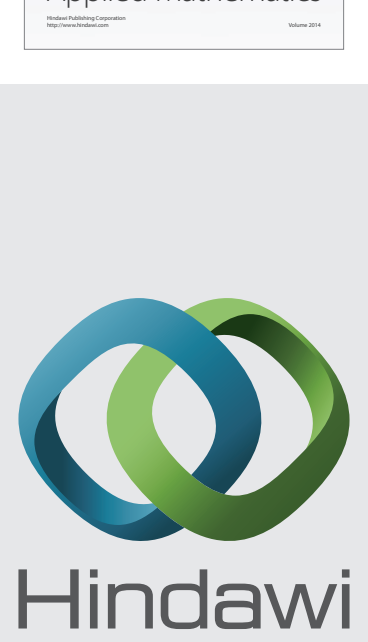

Submit your manuscripts at http://www.hindawi.com
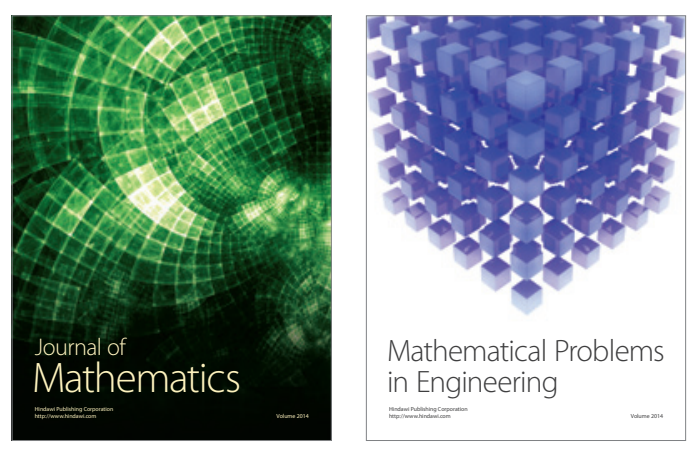

Mathematical Problems in Engineering
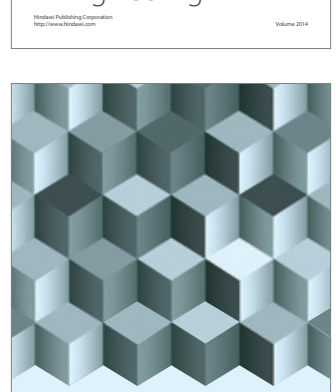

Journal of

Function Spaces
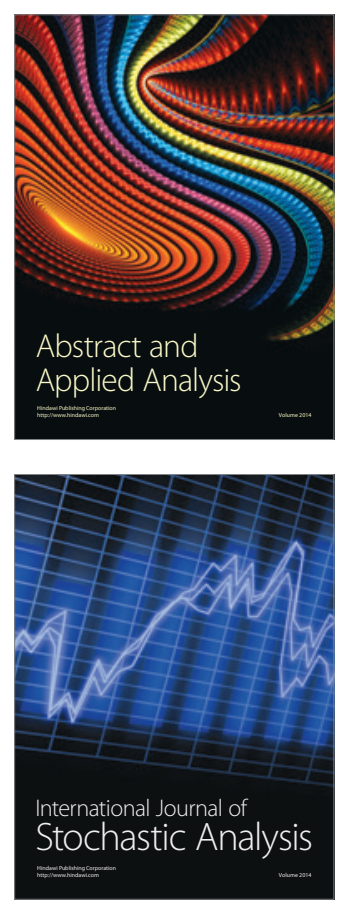

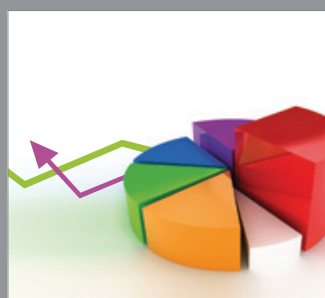

ournal of

Probability and Statistics

Promensencen
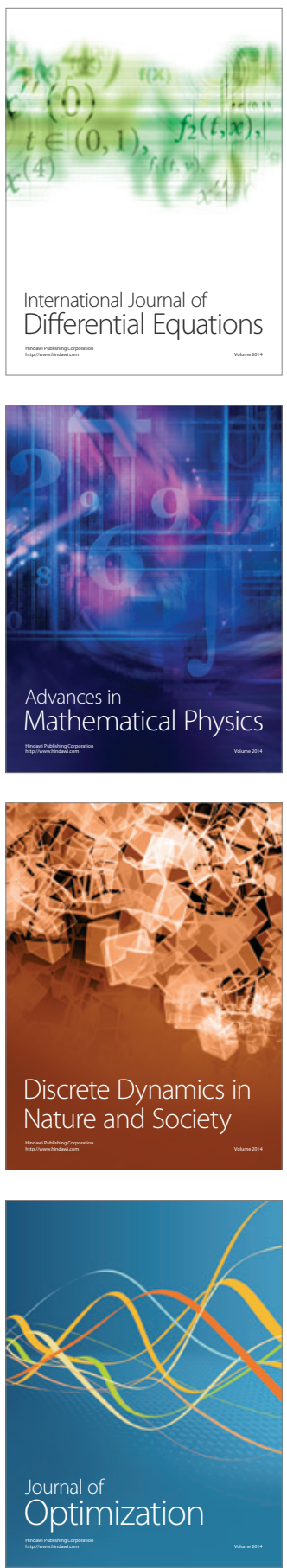